\title{
Deep GMRT 150 MHz observations of LBDS
}

\author{
C. H. Ishwara-Chandra*, S. K. Sirothia, Y. Wadadekar \\ National Centre for Radio Astrophysics, TIFR, P. B. No. 3., Ganeshkhind, Pune 411007, India \\ E-mail: ishwar@ncra.tifr.res.in; sirothia@ncra.tifr.res.in, \\ yogeshencra.tifr.res.in
}

\section{S. Pal}

International Centre for Radio Astronomical Research, University of Western Australia, 35

Stirling Highway, WA, 6009, Australia

E-mail: spal@cyllene.uwa.edu.au

\section{R. Windhorst}

School of Earth \& Space Exploration, Arizona State University P.O. Box 871404 Tempe, AZ 85287-1404, USA

E-mail: Rogier.Windhorsteasu.edu

The most efficient way to search for high redshift radio galaxies is to use its steep radio spectra as high-redshift indicator. Most of the radio galaxies with $\mathrm{z}>3$ have been found using this redshift-spectral index correlation. We have started a programme with the Giant Metrewave Radio Telescope (GMRT) to exploit this correlation at flux density levels about 10 to 100 times deeper than the known high-redshift radio galaxies which were identified primarily using the already available radio catalogues. In our programme, we have obtained deep, high resolution radio observations at $150 \mathrm{MHz}$ with GMRT for several ' deep $^{\prime}$ fields which are well studied at higher radio frequencies and in other bands of the electromagnetic spectrum, with an aim to detect candidate high redshift radio galaxies. In this poster we present results from the deep $150 \mathrm{MHz}$ observations of LBDS-Lynx field, which has been already imaged at 327, 610 and $1412 \mathrm{MHz}$ with the Westerbork Synthesis Radio Telescope (WSRT) and at 1400 and $4860 \mathrm{MHz}$ with the Very Large Array (VLA). The $150 \mathrm{MHz}$ image made with GMRT has a rms noise of $\sim 0.7 \mathrm{mJy} / \mathrm{beam}$ and a resolution of $\sim 19^{\prime \prime} \times 15^{\prime \prime}$. It is the deepest low frequency image of the LBDS-Lynx field. The source catalog of this field at $150 \mathrm{MHz}$ has about 765 sources down to $\sim 20 \%$ of the primary beam response, covering an area of about 15 degree $^{2}$. Spectral index was estimated by cross correlating each source detected at $150 \mathrm{MHz}$ with the available observations at 327, 610, 1400 and $4860 \mathrm{MHz}$ and also using available radio surveys such as WENSS at $327 \mathrm{MHz}$ and NVSS and FIRST at $1400 \mathrm{MHz}$. We find about 150 radio sources with spectra steeper than 1. About twothird of these are not detected in Sloan Digital Sky Survey (SDSS), hence are strong candidate high-redshift radio galaxies, which need to be further explored with deep infra-red imaging and spectroscopy to estimate the redshift.

ISKAF2010 Science Meeting - ISKAF2010

June 10-14, 2010

Assen, the Netherlands

\footnotetext{
* Speaker.
} 


\section{Introduction}

It had been noticed in the early 80 's that the fraction of radio sources that can be optically identified is lower by a factor of 3 or more for steep spectrum radio sources $\left(\alpha>1 ; S_{v} \propto v^{-\alpha}\right.$; Tielens, Miley \& Wills, 1979, Blumenthal \& Miley, 1979; Gopal-Krishna \& Steppe, 1981), suggesting that the radio sources with steeper spectra at decimeter wavelengths are more distant compared to the ones with normal spectra $(1>\alpha>0.5)$. This correlation has been successfully applied to search for radio sources at high redshifts (Rottgering et al., 1994; De Breuck et al. 2000, 2002; Klamer et al. 2006). Until today, about 45 radio galaxies are known beyond redshift of 3 , and nearly all of them were discovered through the radio spectral index - redshift correlation.

Till date, the search for HzRGs using the radio spectral index - redshift correlation was mostly limited by the sensitivity limit of the shallow, wide-area radio surveys. This bias has led to the detection of only the brightest HzRGs at the top end of the radio luminosity function, which is about three orders of magnitude brighter than the luminosities at the lower end of FR-II population (Fanaroff \& Riley, 1974). Deeper observations at low radio frequencies are needed to discover HzRGs that are 10 to 100 times less luminous than most of the known HzRGs, and these could be the high-redshift counterparts of more typical FR-II radio galaxies. The $150 \mathrm{MHz}$ band of GMRT (Giant Metrewave Radio Telescope, India, http://www.ncra.tifr.res.in) with its large field of view (3 degrees), high angular resolution $\left(\sim 20^{\prime \prime}\right)$ and better sensitivity $(\sim 1 \mathrm{mJy}$ from a full synthesis observation) is well suited for this kind of work.

Encouraged by this, we have started a programme to observe several carefully chosen deep fields at $150 \mathrm{MHz}$ with GMRT with an aim to detect steep spectrum radio sources to flux density levels much fainter than that of known high-redshift radio sources. In this paper we present deep $150 \mathrm{MHz}$ radio observations of the LBDS-Lynx field (Windhorst et al. 1984) with the GMRT with the primary aim of detecting steep spectrum radio sources which are candidate HzRGs (C. H. Ishwara-Chandra et al. 2010). In Section 2, we present supporting arguments for reviving the search for HzRGs using the steep spectrum technique. Observations, data analysis are presented in Section 3. Results and discussions are presented in Section 4 and concluding remarks in Section 5.

\section{Re-visit of Ultra-steep spectrum technique for finding HzRGs}

Miley \& de Breuck (2008) in their review have provided a table of all known HzRGs till date with redshift more than 2. In order to understand whether the known HzRGs represent typical FRII radio sources at high-redshifts, or the highest luminosity sources in that category, we plot the observed flux densities at $150 \mathrm{MHz}$ of all known HzRGs ( $>$ > 3), computed using the available flux density measurements and the spectral index (Figure 1). It is clear from the figure that nearly all of the known HzRGs are two to three orders of magnitude more luminous than the FRI/FRII break luminosity. This is largely due to the selection effects because nearly all the searches for steep spectrum radio sources use sky surveys such as WENSS and SUMSS at low radio frequencies and NVSS at higher radio frequencies. The median flux density of all known HzRGs at $z>3$ at 150 $\mathrm{MHz}$ is $\sim 1.3 \mathrm{Jy}$ ( $90 \%$ of them are stronger than $\sim 0.5 \mathrm{Jy}$ ), whereas the FRI/FRII break luminosity is more than two orders of magnitude fainter. 


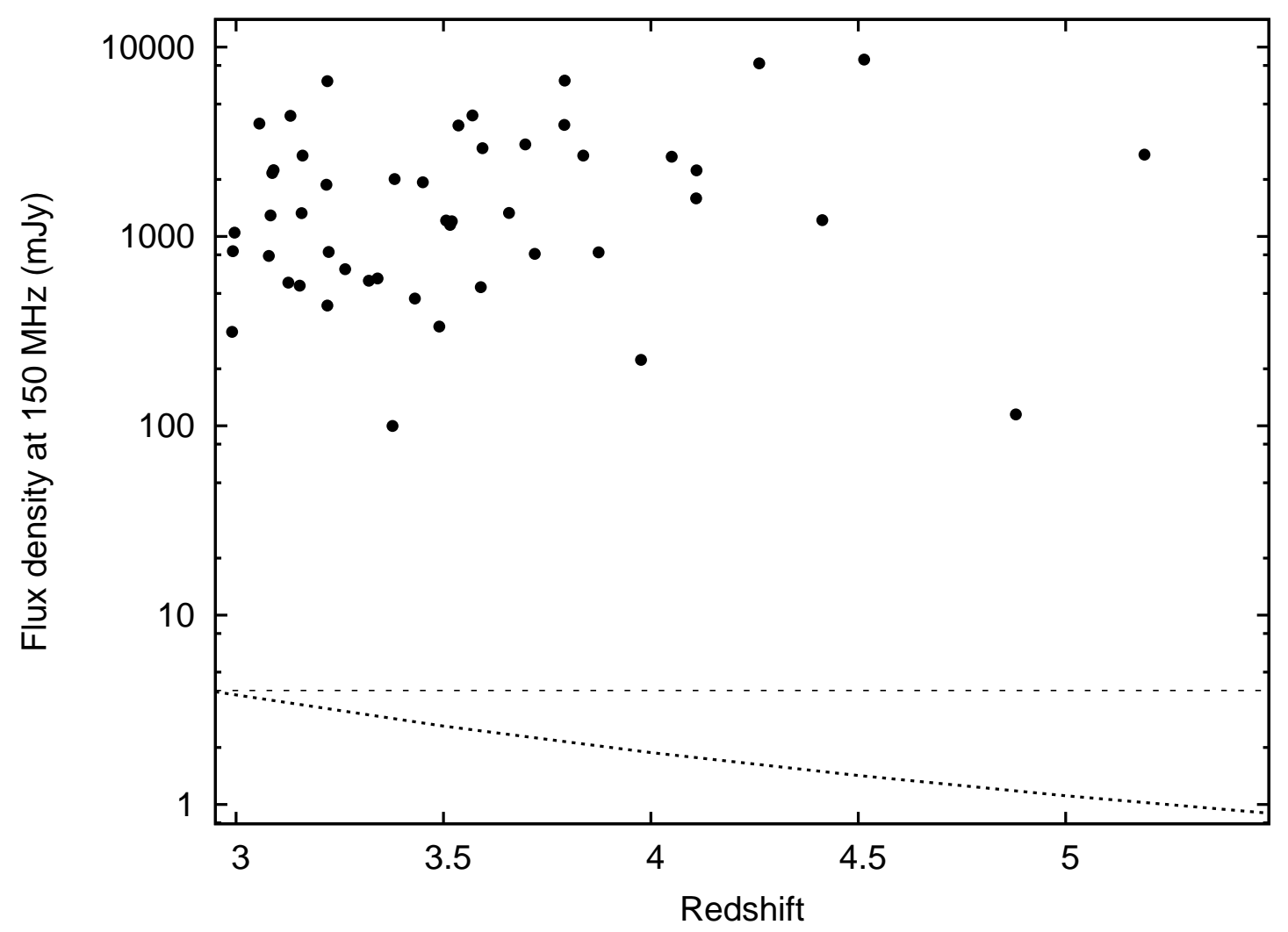

Figure 1: The $150 \mathrm{MHz}$ flux densities of known HzRGs, extrapolated using the available spectral index and flux density measurements. The dotted line at the bottom indicates the observed $150 \mathrm{MHz}$ flux density corresponding to the rest-frame FRI/FRII break luminosity. The dashed horizontal line is the GMRT detection limit from the present $150 \mathrm{MHz}$ observation. It is clear from this figure that a large number 'normal population' of FRIIs, that are 10 to 100 times less luminous than the known HzRGs are yet to be discovered.

The above argument implies that the known HzRGs represent the tip of the ice-berg in luminosity. There are, potentially, a large number of HzRGs yet to be discovered which are expected to be 10 to 100 times less luminous than the known HzRGs (see Fig. 1). Although initially it was believed that there is a 'cut-off' in the radio luminosity function at high-redshift, subsequent to the discovery of several radio galaxies at $\mathrm{z}>4$, this evidence became suspect (Jarvis et al 2001). The radio luminosity function (RLF) of HzRGs indicate that at luminosities 10 to 100 times lower than the known HzRGs, we could expect at least a 10 fold increase in space density of the radio sources beyond the redshift of 3 (Waddington et al. 2001). This large gap, can thus be filled by searching for steep spectrum sources using deep radio observations at low frequencies. The present observation of the LBDS field at $150 \mathrm{MHz}$ with the GMRT has an rms noise of $\sim 0.7 \mathrm{mJy} / \mathrm{beam}$, and the source catalog has about 750 sources having a flux density of $\gtrsim 4 \mathrm{mJy}$ (C. H. Ishwara-Chandra et al. 2010). Using our observations, we have obtained about 100 radio sources with spectral index steeper than 1 having no optical counterparts in the SDSS. The median $150 \mathrm{MHz}$ flux density for these sources is $\sim 110 \mathrm{mJy}$, which is an order of magnitude fainter than the median flux density at $150 \mathrm{MHz}$ for known HzRGs. 


\section{Observation and data analysis}

The $150 \mathrm{MHz}$ observations of the LBDS-Lynx field centered at RA of 08h41m46s and DEC of $+44 \mathrm{~d} 46^{\prime} 50^{\prime \prime}$ (J2000) were carried out with the Giant Metrewave Radio Telescope (GMRT) on December 11, 2006 using a bandwidth of $16 \mathrm{MHz}$. The GMRT consists of 30 antennas, each of 45 meter diameter located $90 \mathrm{~km}$ from Pune, India and operates at five frequency bands from $150 \mathrm{MHz}$ to $1450 \mathrm{MHz}$ (Swarup et al. 1991). The data were analysed using AIP S++ (v1.9; build \#1556; see Sirothia 2008, Sirothia et al. 2009). The final image was made after several rounds of phase selfcalibration, and one round of amplitude-and-phase self-calibration. From the estimate of median spectral index of the sources in the field (0.78), which was close to the expected value, we infer that the error in the flux density scale is $\lesssim 10 \%$ at $150 \mathrm{MHz}$. The final rms noise achieved at 150 $\mathrm{MHz}$ near the center of the field in a source free region was $\sim 0.7 \mathrm{mJy} / \mathrm{beam}$ with a resolution of $\sim 19^{\prime \prime} \times 15^{\prime \prime}$ at position angle of $27^{\circ}$.

Deep radio observations of this field are available at 327, 610 and $1412 \mathrm{MHz}$ using the WSRT and at 1400 and $4860 \mathrm{MHz}$ with the VLA. However, all these observations combined cover less than $50 \%$ of the GMRT $150 \mathrm{MHz}$ area. We also use the WENSS data at $327 \mathrm{MHz}$ and NVSS and FIRST data at $1400 \mathrm{MHz}$ for the entire field.

\section{Results and discussion}

\subsection{The 150 MHz source catalog and spectral index estimates}

A catalogue of sources out to the $20 \%$ of the peak primary beam response was created with the peak source brightness greater than 6 times the local rms noise value. The details of the source extraction criterion are given in Sirothia et al. (2009). The final catalogue contains about 765 sources above a flux density limit of $\sim 4$ mJy. The majority (about two-third) of the sources are unresolved within the resolution of the observation.

The counterparts for the $150 \mathrm{MHz}$ sources at higher radio frequencies were searched within a $20^{\prime \prime}$ radius from the $150 \mathrm{MHz}$ position. In addition to the published deep observations of the LBDS field at 327, 610, 1412, 1462 and $4860 \mathrm{MHz}$, we used the WENSS catalog at $325 \mathrm{MHz}$ and the NVSS and FIRST catalogs at $1400 \mathrm{MHz}$ to obtain the spectral index of sources found at $150 \mathrm{MHz}$. The typical error on the spectral index estimate was about 0.1, or better. A small fraction of the sources (3\%) do not fit well with a straight spectrum and these are mostly sources with spectral turnover. The spectral index distribution is presented in Fig. 2.

A total of 639 sources out of $765(83 \%)$ have spectral index determined. The median spectral index of the sample is 0.78 , which is in good agreement with the similar measurements available in the literature. Our sample of steep spectrum sources consists of 157 sources with spectra steeper than 1 (and not 1.3 as used by several authors earlier) for the reasons discussed in detail in IshwaraChandra et al. 2010.

\subsection{Sample of steep spectrum sources}

We have inspected the spectra where three or more frequency measurements are available for signatures of spectral curvature. There is no clear indication of spectral curvature in most of the sources. We have cross matched the sample of 157 sources, with spectral index steeper than 1 with 


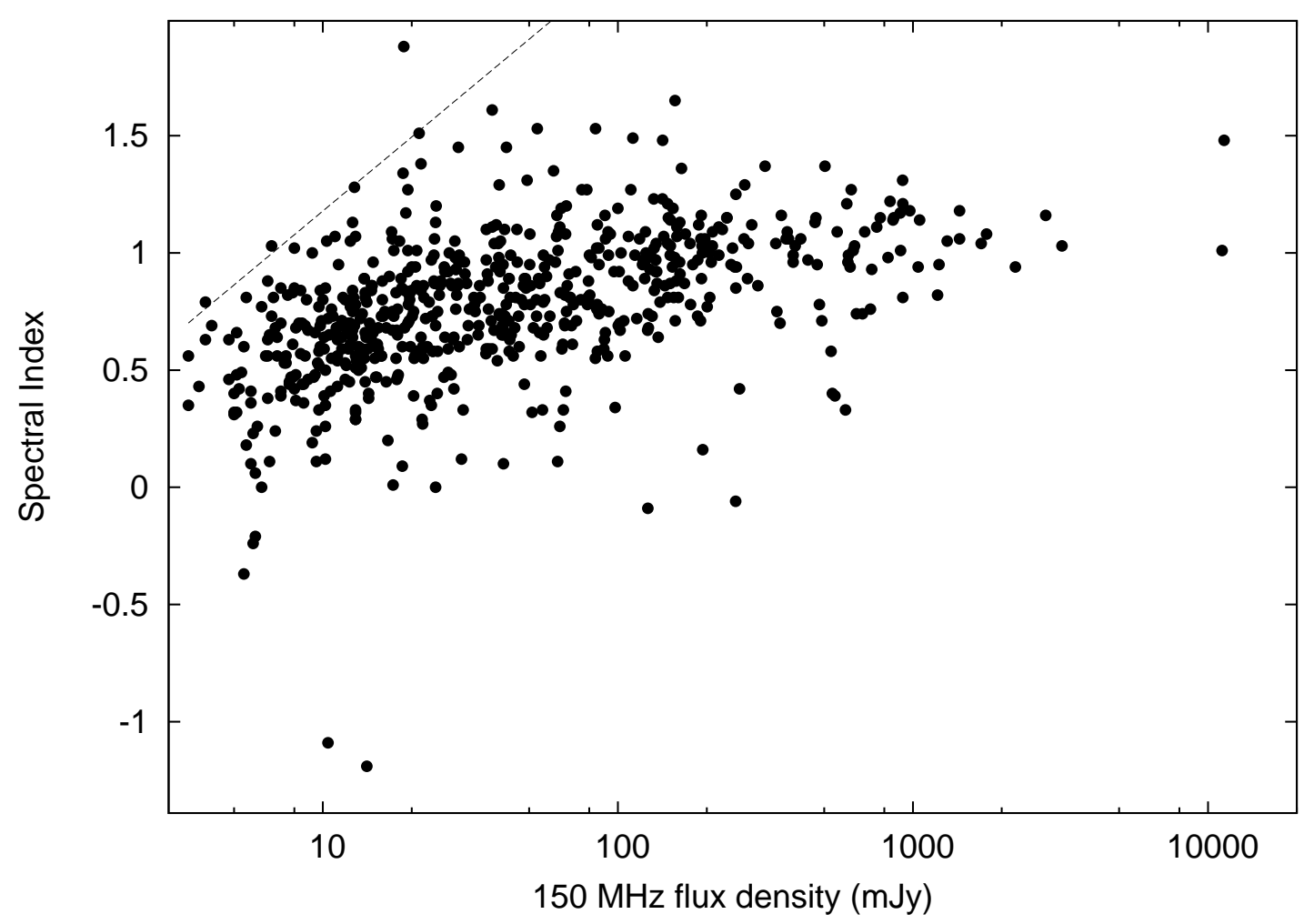

Figure 2: The spectral index distribution of sources detected at $150 \mathrm{MHz}\left(\mathrm{S}_{v} \propto v^{-\alpha}\right)$. The spectral index was computed whenever a counterpart was found at $610 \mathrm{MHz}$ or higher frequencies, irrespective of detection at $325 \mathrm{MHz}$. The dashed line shows the spectral index limit corresponding to five sigma limit of FIRST. There is a weak trend indicating that the fainter sources tend to have flatter spectra.

VLA FIRST survey. If the counterpart is seen in FIRST, we have taken the position from FIRST survey to obtain better accuracy. Among the 157 sources, 8 sources did not have counterparts in FIRST and hence the $150 \mathrm{MHz}$ position was used to cross match with SDSS.

We positionally matched each steep spectrum source position with the photometric object catalog (PhotoObjAll) from the most recent (DR7) release of the Sloan Digital Sky Survey (SDSS; Abazajian et al. 2009). 59 radio sources from this sample had at least one SDSS primary object within 6 arcsec (38\% identification). The remaining 98 sources had no optical counterpart in SDSS within $6^{\prime \prime}$ radius. The median $150 \mathrm{MHz}$ flux density for the sources not having counterpart in SDSS is $\sim 110 \mathrm{mJy}$, which is more than an order of magnitude fainter than the median flux density at $150 \mathrm{MHz}$ for known HzRGs (section 2). One of the steep spectrum source without SDSS counterpart (GMRTJ084533+455835), is unresolved at $150 \mathrm{MHz}$, but resolves into a clear compact FRII source of about 8 arcsec size in VLA FIRST. Using the $150 \mathrm{MHz}$ flux density and FRI/FRII break luminosity, we estimate that this source should be at a redshift of $\sim 2$ or higher (for its luminosity to be above the FRI/FRII break).

\section{Concluding Remarks}

We have initiated a major programme to search for steep spectrum radio sources with the GMRT with the aim to detect high-redshift radio galaxies of moderate luminosity. The fields for 
this programme are carefully chosen such that extensive data already exist at higher radio frequencies and deep optical imaging and/or spectroscopy is also available for most of the fields. Here we have presented the results from deep $150 \mathrm{MHz}$ low frequency radio observations of the LBDS-Lynx field with the Giant Metrewave Radio Telescope (GMRT), India reaching an rms noise of of $\sim 0.7$ $\mathrm{mJy} / \mathrm{beam}$ and a resolution of $\sim 20^{\prime \prime}$. We have demonstrated that this GMRT programme can search for high-redshift radio galaxies more than an order of magnitude fainter in luminosity compared to most of the known HzRGs. We provide a sample of about 100 candidate HzRGs with spectral index steeper than 1 and no optical counterpart to the SDSS sensitivity limits. A significant fraction of the sources are compact, and are strong candidates for high-redshift radio galaxies. These sources will need to be followed up at optical and near-infrared bands to estimate their redshifts.

\section{Acknowledgments}

GMRT is operated by the National Centre for Radio Astrophysics of the Tata Institute of Fundamental Research. This research has made use of the NASA/IPAC Extragalactic Database (NED) and Sloan Digital Sky Survey (SDSS http://www.sdss.org/).

\section{References}

[] Abazajian K. N. et al., 2009, ApJS, 182, 543

[] Blumenthal G., Miley G., 1979, A\&A, 18, 13

[] De Breuck C., van Breugel W., RÃúttgering H. J. A., Miley G., 2000, A\&AS, 143, 303.

[] De Breuck C., van Breugel W., Stanford S. A., RÃúttgering H., Miley G., Stern D., 2002, AJ, 123, 637

[] Fanaroff B. L., and Riley J. M, 1974, MNRAS, 167P, 31

[] Gopal-Krishna., Steppe, H., 1981, A\&A, 101, 315

[] Ishwara-Chandra, C. H., Sirothia, S. K., Wadadekar, Y., Pal, S., Windhorst, R., 2010, MNRAS, 405, 436

[] Jarvis M. J., Rawlings S., Willott C. J., Blundell K. M., Eales S., Lacy M., 2001, MNRAS, 327, 907

[] Klamer I. J., Ekers R. D., Bryant J. J., Hunstead R. W., Sadler E. M., De Breuck C., 2006, MNRAS, 371,852

[] Miley G, K., de Breuck C., 2008, A\&ARv, 15, 67

[] Rottgering H. J. A., Lacy M., Miley G. K., Chambers K. C., Saunders R., 1994, A\&AS, 108, 79

[] Sirothia S. K., 2008, PhD Thesis, TIFR.

[] Sirothia S. K., Saikia D. J., Ishwara-Chandra C. H., and Kantharia N. G., 2009, MNRAS, 392, 1403

[] Swarup G., Ananthakrishnan S., Kapahi V. K., Rao A. P., Subrahmanya C. R., Kulkarni V. K., 1991, Current Science, 60, 95.

[] Tielens A. G. G. M., Miley G. K., Willis A. G., 1979, A\&AS, 35, 153.

[] Waddington I., Dunlop J. S., Peacock J. A., Windhorst R. A., 2001, MNRAS, 328, 882

[] Windhorst R. A., van Heerde G. M., Katgert P., 1984, A\&AS, 58, 1 